\title{
An Impulse Response Flow Cytometric Technique for Blood Cell Characterisation -- Instrumentation and Preliminary Evaluation
}

\author{
Y Liu, E W Abel *, J J F Belch **, S Chen \\ Department of Electrical and Electronic Engineering, University of Portsmouth, Portsmouth POI 3DJ, UK \\ School of Biomedical Engineering, University of Dundee, Dundee DD1 4HN, UK * \\ Department of Medicine, University of Dundee, Dundee, DD1 9SY, UK ** \\ E-mail: yingl@ee.port.ac.uk
}

\begin{abstract}
A new technique for studying the properties of blood and other cells has been developed. It is based on measurement of the continuous electrical impedance spectrum of individual cells. The cellular impedance is obtained by applying an impulse and recording the impulse response while a stream of cells suspended in an electrolyte pass through a measurement aperture in a single file. Preliminary evaluation has demonstrated that the instrument prototype is capable of detecting characteristic spectra of different cell populations. This paper presents some theoretical aspects of the technique, an outline of the instrumentation design and preliminary experimental results.
\end{abstract}

\section{Key Words}

Cellular Impedance, Cytometry, Impulse Response

\section{Introduction}

Vascular disease and cancers are responsible for a greater mortality than any other disease in the western world. Early diagnosis and treatment can save not only lives but also health care resources. In many cases, patients with risk factors for developing vascular disease and cancers have abnormal blood cell behaviour [1,2,3,4]. Cell abnormalities may be characterised by altered size, morphology, chemical composition and responses to chemical agents, however measurement of the electrical characteristics of biological cells has proved to be an effective tool in determining cell behaviour and activity.

Changes in cell electrical characteristics have been shown to reflect pathological conditions [5,6]. Techniques for DC cellular impedance measurement are well established (e.g. Coulter flow cytometer systems) and are used extensively in haematology laboratories to differentiate cell populations of different sizes. However, cell discrimination and classification based on size alone are insufficient for diagnostic applications, as cells do not necessarily alter in size in disease. Additional discriminators are needed based on measurements of the properties of the cellular structures such as the membrane, nucleus and cytoplasm. Measurement of cellular impedance over a range of frequencies has been explored by a number of researchers in the hope of establishing links between the impedance spectrum and cellular structure, so that the measurement of the impedance spectrum can be used as diagnostic tool $[7,8,9,10]$.

The techniques used so far can be generally categarised into single cell measurement (represented by the micropipet method) and bulk cell measurement (represented by the micro-filter embedded method and bulk suspension impedance measurement methods). The micropipet technique is a static cellular impedance measurement technique, in which a single cell is manipulated by a micropipet (which also serves as a micro-electrode) and pressed on or through the cell membrane. This allows ample time for detailed impedance measurement [11]. The potential of the micropipet technique as a clinical diagnostic tool has been hampered by its poor high frequency response and particularly by the requirement of microscopic manipulation of cells. Schmulker [12] reported a transient response technique, with the use of a polycarbonate micro-filter in which the cells become embedded. This technique eliminates the requirement of cell manipulation but is unable to obtain measurements on large numbers of indivadual cells.

These and other previous efforts have had very limited success in clinical applications as none of the techniques is able to fulfil two clinically important criteria; firstly, the capability of providing a continuous impedance spectrum of an individual cell rather than at a few discrete frequencies only, and secondly, the provision of a means of rapid and automated analysis, allowing large number of cells to be processed in order to yield statistically meaningful results.

We have developed a technique based on the measurement of the continuous cellular impedance spectrum using an 
electrical impulse response method. It is capable of measuring continuous impedance spectrum of individual cells in suspension. The instrument is incorporated into a flow cytometer system allowing larger number of cells to be measured in an automated manner. This paper presents some theoretical aspects of the technique, the instrumentation used and results of a preliminary investigation to test the capability of the instrument to discriminate cells of different types.

\section{Principle of Operation and Instrumentation}

The basis of this technique is to probe an individual cell with a very short current pulse (about $100 \mathrm{~ns}$ ) containing all frequency components within the measurable bandwidth of the system. The pulse response of the system, which is defined as consisting of the aperture and the cell passing through the aperture, is formed from the convolution of the input with the system function. The impulse function of the exciting signal theoretically contains all frequencies (although in practice it is necessarily band limited) so the output is equal to the system function. Its frequency spectrum therefore contains information about cellular characteristics which, if the instrument has sufficient resolution and sensitivity, could differentiate normal and disease conditions. Combining this technique with flow cytometric methods enables thousands of cells in a sample to be measured in just a few seconds.

As the pulse width of impulse applied (typically 50ns $250 \mathrm{~ns}$ ) is negligible compared to the time during which the cell remains in the aperture (typically 30-60 $\mu \mathrm{s}$ ), the cells are effectively stationary during measurement. The process can therefore be considered to be a problem of static system identification. Thus,

$$
y(t)=\int x(t) \bullet h(t-\tau) d \tau
$$

where $y(t)$ is the system time domain output, $x(t)$ is the pulse input and $h(t-\tau)$ is the system pulse response transfer function (as $\tau$ varies from plus/minus infinity (get correct symbols)), which is determined by the electrical characteristics of the measurement aperture and the cell.

This relationship is more conveniently expressed in frequency domain as,

$$
Y(j \omega)=X(j \omega) \bullet H(j \omega)
$$

where $Y(j \omega), X(j \omega)$ and $H(j \omega)$ are the transfer functions of $y(t), x(t)$ and $h(t)$ respectively.

The system transfer function $H(j \omega)$ can be further expressed in

$$
H(j \omega)=H_{A}(j \omega) H_{C}(j \omega)
$$

where $H_{A}(j \omega)$ and $H_{C}(j \omega)$ represent the frequency response of the measurement aperture and the cell respectively..

As the transfer functions of the input $X(j \omega)$ and the aperture $\mathrm{H}_{\mathrm{A}}(\mathrm{j} \omega)$ can be determined experimentally, once the frequency domain expression of the system $Y(j \omega)$ is obtained, it is possible to identify the cell transfer function $\mathrm{H}_{\mathrm{C}}(\mathrm{j} \omega)$. That is,

$$
\begin{aligned}
& Y(j \omega)=X(j \omega) \bullet H_{A}(j \omega) \bullet H_{C}(j \omega) \\
& H_{C}(j \omega)=H_{A}^{-1}(j \omega) \bullet X^{-1}(j \omega) \bullet Y(j \omega)
\end{aligned}
$$

Even if the responses of the cell $\mathrm{H}_{\mathrm{C}}(\mathrm{j} \omega)$, the response of the aperture $H_{A}(j \omega)$ and the pulse transfer function $X(j \omega)$ can not be deconvolved, the system response $Y(j \omega)$ as a whole can be used as the indicative characteristic the cell as long as $\mathrm{H}_{\mathrm{A}}(\mathrm{j} \omega)$ remains constant during entire period of measurement.

The instrument system is schematically shown in Figure 1. Cells in suspension are delivered to a measurement flow chamber by a motor driven syringe. The sample flow is escorted by a sheath flow through the centre of a $100 \mu \mathrm{m}$ diameter aperture under hydrodynamic focusing. This aperture in the measurement flow chamber forms the sensing zone of the instrument. A classical four-electrode arrangement is applied for pulsing and sensing. The flow chamber has a characteristic impedance, dominated by the size and shape of the aperture and the electrolyte. A low level DC current is applied to the pulse generating electrodes. The presence of a cell in the aperture causes a small change in impedance. This change is sensed by a detector which in turn fires a current pulse though the electrodes. The response is detected with an ultra-low noise wideband differential amplifier.

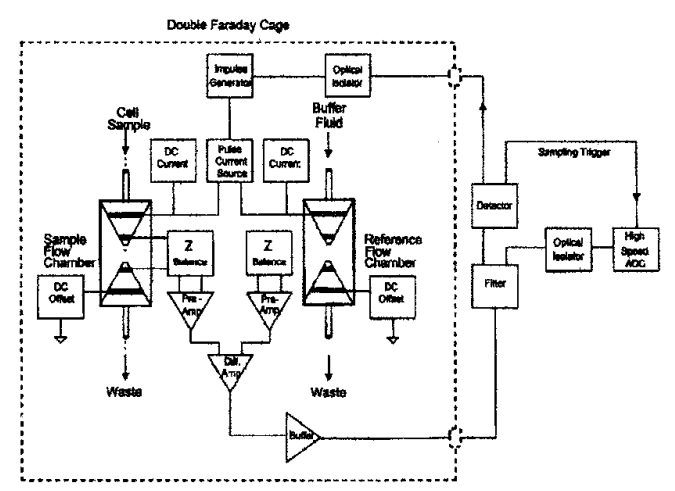

Figure 1 IRIS system schematic

A reference flow chamber, which has identical electrical characteristics with the sensing flow chamber, is used to subtract the pulse response due to aperture impedance. In order to match the electrical characteristics of the two flow 
chambers over a frequency range, tunable $\mathrm{RC}$ networks are used to fine tune the response of the flow chambers. The amplified signal is optically isolated and directed to a $100 \mathrm{MHz}$ computer based data acquisition system recorded on the computer's hard disc. All transducers, amplifiers and other electronics are shielded using a double layered Faraday cage to minimise electromagnetic interference.

Although the principle of the measurement is straightforward, the design and implementation of the instrument have proven to be technically challenging, in particular the high performance amplifier system. This amplifier must function in a very difficult environment extracting wide band, microvolt signals.

A custom designed low noise wide band amplifier system comprising two front-end pre-amplifiers and a differential amplifier has been developed. The pre-amplifier module is implemented using a wide band operational amplifier (AD829, Analog Devices) with a FET/Bipolar transistor extension at its inputs. The design employs a global current feedback topology which combines the transistors and the operational amplifier in an integrated system. The high open loop gain provides a maximal flat bandwidth and ensures low noise figure for the amplifier. The FET/Bipolar transistor input extension is optimised for high input impedance, high slew rate and high common mode input range. The design uses local series/parallel feedback, an active current source and cascade techniques that are frequently used in modern operational amplifier design. The differential amplifier is a customised video amplifier system which operates at high speed and has a high common mode rejection ratio.

\section{Preliminary Evaluation}

The preliminary evaluation of the system described here is aimed to demonstrate that a continuous frequency spectrum representing the unique signature characteristics of a cell type can be obtained with the impulse response flow cytometer prototype and the characteristic spectra of different cell populations can be discriminated.

The samples used in the studies ware latex calibration particles (size E34, Coulter Electronics, Luton, UK) and cultured cell lines. The latex particles are solid spherical test micro-particles, about $10 \mu \mathrm{m}$ in diameter, which have a well defined electrical characteristic and matches well with the single shell cell model used in previours cell impeadance studies $(7,8,12)$. The cell line used was a homogeneous sample of cells grown from a few identical white blood cells. This uniform population is ideally suited for the purpose of evaluating the instrument. Two cell lines were used in this investigation. These are $\mathrm{T}$ cell line and $B$ cell line. The $T$ cells are leukaemic cells and self-perpetuating, while the $B$ cells are lymphocytes and modified using Epstein-Barr (EB) virus to achieve the necessary immortality. The cells were suspended in a physiological electrolyte buffer (Isonton II, Coulter Electronics Ltd., Luton, UK). All the test samples were diluted to achieve $400-600$ counts per second.

The aperture was initially flushed with Isoton II for several minutes to stabilise the instrument and to remove any residual material from previous tests, thereby minimising cross-contamination. . This procedure was carried out after each test. The pulse response signals were digitised using an 8-bit $100 \mathrm{MHz}$ data acquisition system (Signatec Inc, Corona, CA, USA). The data were processed off-line. The signals were converted into a frequency spectrum representation using a Cooley-Tukey FFT routine, to avoid the need for time domain processing of the complex impulse response signals. The spectra of all the individual cells in the sample were superimposed to form a scattered spectrum distribution. This is further to develop a mean spectrum of population representation. The mean spectrum of the sample is used as the characteristic signature and comparing the mean spectra from different cell populations allows discrimination to be made on an qualitative basis.

The preliminary tests show E34 latex particles have strong resonance at $0.7 \mathrm{MHz}, 2.3 \mathrm{MHz}$ and $6.6 \mathrm{MHz}$. The T cells have resonance at $1.0 \mathrm{MHz}, 2.9 \mathrm{MHz}, 3.6 \mathrm{MHz}, 4.5 \mathrm{MHz}$, $5.5 \mathrm{MHz}$ and $6.3 \mathrm{MHz}$ while the $\mathrm{B}$ cells at $0.6 \mathrm{MHz}$, 1.7 MHz, 4.3MHz, $5.0 \mathrm{MHz}, 5.8 \mathrm{MHz}$ and $6.3 \mathrm{MHz}$.

\section{References}

1. BELCH, J.J.F, 1990. The role of the white blood cell in arterial disease. Blood Coag. and Fibrinolysis 1, 183-192.

2. LAU, C., O'DOWD, A., BELCH, J.J.F. 1992. White cell activation in the Raynauds Phenomenon of systemic sclerosis and vibration induced white finger syndrome. Ann. Rhem. Dis. $51(2), 249-252$

3. BRIDGES, A.B., MLAREN, M., SCOTT, N., SANIABADI, A., BELCH, J.J.F., 1991. The relationship of oxidative stress to thrombotic tendency in type 1 diabetic patients with retinopathy. Diab. Med. 8, 860-865.

4. MITCHELL, H., PENTYCROSS, C.R. ABEL,
E.W., WOOD, P., BAGSHAWE, K.D.B., 1980. The SCM test for cancer, an evaluation in terms of lymphyocytes from healthy donors and cancer patients. Br. J. Cancer 41 (5), 772-777.

5. PAULY, H. 1959. Electrical Conductance and Dielectric Constant of the Interior of Erythrocytes. Nature 183, 333-334.

6. CHANARIN, I., BROZOVIC, M., TIDMARSH, E., WATERS, D.A.W. 1984. Blood and its diseases, 3rd edn. Churchill Livingstone, Edinburgh.

7. SCHWAN, H.P. 1959. Alternating Current Spectroscopy of Biological Substances. Proceedings of the IRE. 47, 1841-1855. 
8. PAULY, H., SCHWAN, H.P. 1966. Dielectric Properties and Ion Mobility in Erythrocytes. Biophys J. 6, 621-639.

9. SCHMUKLER, R, PILLA, A., CERF, G., LEE, M. 1982. A new transient technique for measurement of isolated cell impedance. Proc 10th N.E Bioenging. Conf. IEEE Press 213-216.

10. COULTER, W.H., HOGG, W.R. 1970. Signal modulated apparatus for generating and detecting resistive and reactive changes in a modulated current path for particle classification and analysis. U.S Patent, 3,502,974.

11. TAKASHIMA $S$ et al., Frequency domain studies of impedancy characteristics of biological cells using micropipet technique, J. Biophys., 54, 1988.

12. SCHMUKLER, R, PLLA, A., CERF, G., LEE, M. 1982. A new transient technique for measurement of isolated cell impedance. Proc 10th N.E Bioenging. Conf. IEEE Press $2 \overline{13-2} 16$. 